\title{
Cosmic ray study by means of reflected EAS Cherenkov light method with the SPHERE-2 detector
}

\author{
D.A. Podgrudkov ${ }^{* a d}$, R.A. Antonov ${ }^{a}$, E.A. Bonvech ${ }^{a}$, D.V. Chernov ${ }^{a}$, Miroslav \\ Finger $^{b c}$, Michael Finger ${ }^{b c}$, T.A. Dzhatdoev ${ }^{a}$ \\ a Skobeltsyn Institute of Nuclear Physics, Lomonosov Moscow State University, Leninskie gory \\ 1-2, 119991, Moscow, Russia \\ ${ }^{b}$ Faculty of Mathematics and Physics, Charles University, Ovocny trh 3-5, 116 36, Prague 1, \\ Czech Republic \\ ${ }^{c}$ Joint Institute for Nuclear Research, Joliot-Curie 6, 141980 Dubna, Moscow region, Russia \\ ${ }^{d}$ Faculty of Physics, Lomonosov Moscow State University, Leninskie gory 1-2, 119991, Moscow, \\ Russia \\ E-mail: timur1606@gmail.com, antr@dec1.sinp.msu.ru, \\ bonvech@yandex.ru, chredecl.sinp.msu.ru, miroslav.fingerecern.ch, \\ michael.fingerecern.ch, d.a.podgrudkovaphysics.msu.ru
}

\begin{abstract}
The approach to cosmic ray (CR) study with reflected optical Vavilov-Cherenkov radiation (Cherenkov light) was proposed long ago. At present the SPHERE-2 detector is the only existing apparatus that have detected a significant sample of extensive air showers (EAS) by means of this method. At the same time the recorded data allows detailed reconstruction of EAS lateral distribution function (LDF) used to study primary CR mass composition.

We report on the status and results of the SPHERE experiment with the emphasis on the peculiarities of the reflected Cherenkov light technique. Detector response simulation was performed by means of full direct Monte Carlo simulation with account of realistic background and noise patterns recorded during the observational runs. Instrumental acceptance was simulated for various energies, charge numbers and zenith angles of primary nuclei.

Primary energy of observed showers was estimated with a typical statistical uncertainty 10-20\% depending on the primary nucleus parameters. The typical systematic uncertainty of the estimated energy vs the primary charge number was found to be below $3 \%$. The primary all-nuclei spectrum was reconstructed. The fraction of light nuclei vs energy in the energy range 10-100 PeV was estimated by means of an event-by-event approach using the LDF steepness parameter.
\end{abstract}

35th International Cosmic Ray Conference - ICRC2017

10-20 July, 2017

Bexco, Busan, Korea

\footnotetext{
* Speaker.
} 


\section{Introduction}

Modern extensive air shower (EAS) arrays are sophisticated installations that utilize a grid of many dozens or even hundreds coherently working separate detector units spread over a considerable area (up to $3000 \mathrm{~km}^{2}$ [1]). Another experimental approach is to use a compact apparatus lifted over the ground registering optical radiations of EAS. The idea to detect EAS Cherenkov light (CL) reflected from the Earth surface belongs to Prof. A.E. Chudakov [2]. The SPHERE experiment with the SPHERE-2 detector [3] is the ultimate realisation of this idea. In the present paper we discuss the status and results of the SPHERE experiment.

\section{The detector and observations}

The SPHERE-2 detector contained a spherical $1.5 \mathrm{~m}$ diameter mirror, a photomultiplier (PMT) mosaic installed near the focal surface of the mirror, and an aperture diaphragm. The scheme of the detector's optical part is shown in figure 1. During the 2008-2011 runs the data aquisiton systems analogue-to-digital converters (ADCs) time sampling step was set to $25 \mathrm{~ns}$. During the remaining flights all the flights of the 2012-2013 runs ADCs were set to 12.5 ns steps. The SPHERE-2 detector was lifted over the snow-covered surface of Lake Baikal by the BAPA tethered balloon (see figure 2) and registered reflected CL from EAS. The total observation time during the 20082013 runs was 139 hours. The altitude varied from $300 \mathrm{~m}$ to $900 \mathrm{~m}$ above the surface. In total, more than 33 thousand trigger events were recorded.

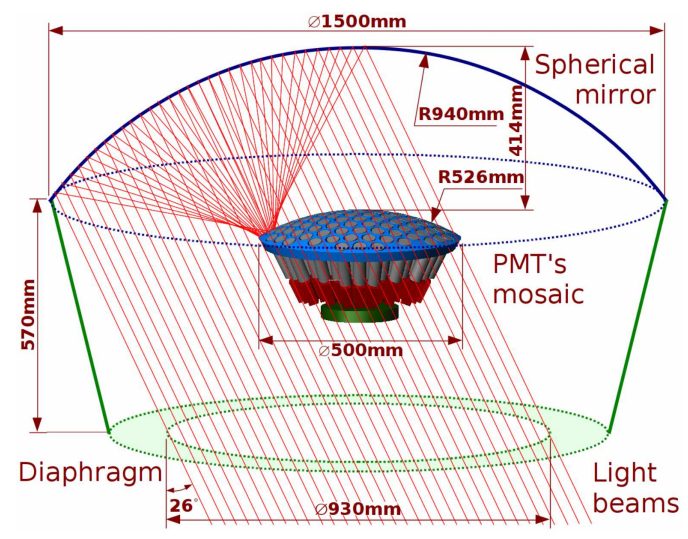

Figure 1: The optical scheme of the SPHERE-2 telescope detector.

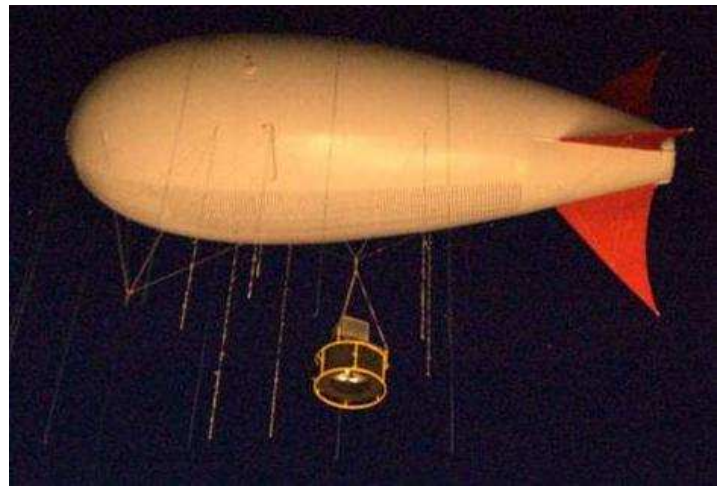

Figure 2: The balloon with the detector being lifted before the observation run.

\section{Low level data analysis}

Most of the trigger events were caused by various backgrounds, including terrestrial light sources and electronics noise. Starting the 2012 season every trigger event was followed by a separate calibration event caused by a flash of the light emitting diode system. The relative calibration procedure is described in [4]. We developed a dedicated procedure for the search of events caused by reflected EAS CL consisting of the following steps: 
1. We checked that the instrumental trigger time is between 220 and 280 time bins and removed all events where this condition is not fulfilled. This criterion is based on the consideration that the instrumental time delay is 230 time bins.

2. Another selection criterion is based on the total time duration $D_{t o t}$ of the event. $D_{t o t}$ equals to full width at half amplitude of the event's "lightcurve" $S(t)=\sum_{i=1}^{109} F(i, t)$ calculated over all 109 measurement channels, where $t$ is the time bin number, $i$ is the channel number, and $F(i, t)$ is the pedestal-subtracted measured signal. $D_{t o t}$ greater than 5 time bins and smaller than 200 time bins was required for an EAS-like event.

3. Finally, we applied several more sophisticated criteria based on the event parameters calculated after the shower front finding procedure. The overall typical efficiency of EAS selection was estimated to be $>98-99 \%$. A total of 1080 events was identified as EAS-like events. For each EAS-like event the direction $\left(\theta_{0}, \phi_{0}\right)$, and the axis coordinates $\left(x_{0}, y_{0}\right)$ were reconstructed.

\section{Detector response simulation}

Using the CORSIKA code [5] with the QGSJET-I high energy hadronic model [6] and the GHEISHA low energy hadronic model [7], we carried out full direct Monte Carlo (MC) simulations of EAS development for a wide range of the primary nucleus parameters: primary energy $E_{M C}$ (from 10 to $100 \mathrm{PeV}$ ), type (proton, helium, nitrogen, iron) and zenith angle (from 0 to $40^{\circ}$ ), and several observation altitudes $H(400,500,580,700,900 \mathrm{~m})$, obtaining in total about 2000 CORSIKA model showers. After that, detector response simulation was performed using a separate Geant 4 code [8] application. Every CORSIKA shower was used multiple times with different axis coordinates, which were uniformly distributed over a $1.5 \cdot H \times 1.5 \cdot H$ square. Finally, a database of model showers (in total $\sim 3 \cdot 10^{5}$ detector response events) was obtained.

Using this latter database, we computed lateral distribution functions (LDF), in units of photoelectrons (ph. el.) arriving at the PMT mosaic. Two examples of such LDF for showers with energies 10 and $100 \mathrm{PeV}$ for the case of $H=400 \mathrm{~m}$ are shown in figure 3 as black and green cir-

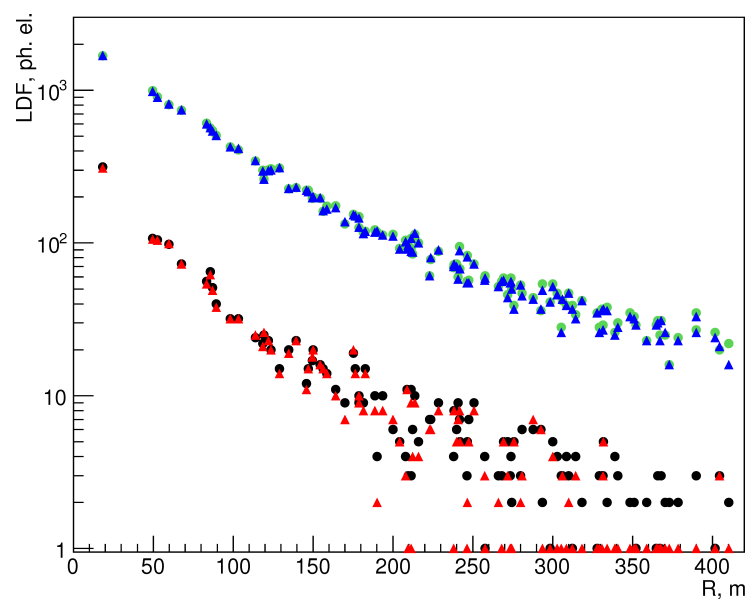

Figure 3: Model LDF before (circles) and after (triangles) digitization procedure for showers with energies $E_{o}=10$ and $100 \mathrm{PeV}$.

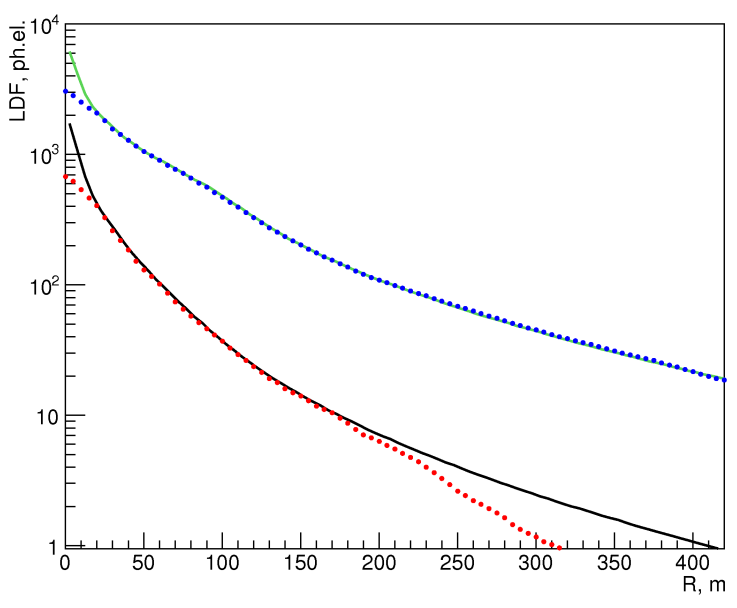

Figure 4: Two examples of composite model LDF (circles) and initial LDF (curves) for showers with energies $E_{0}=10 \mathrm{PeV}$ and $100 \mathrm{PeV}$. 
cles, respectively. At the next step of the simulation, the ADC digitization procedure was applied. The same LDFs after the digitization procedure, assuming $1 \mathrm{ph}$. el. in the code init, are shown in figure 3 for the same showers as red triangles and blue triangles, respectively. Here we added the piedestal 1.0 ph.el. to make the zero values visible.

To reconstruct the axis position, it is convenient to have a large sample of model LDF. Such individual LDFs, however, suffer from large statistical fluctuations due to small number of photons reaching the PMT mosaic. Therefore, we made averaging over 100 responses that originate from the same CORSIKA model shower to compute the so-called "composite" response LDF (CRLDF) that reflects the imprint of the SPHERE-2 detector, but have statistical fluctuations suppressed. Two examples of CRLDF for the same $10 \mathrm{PeV}$ and $100 \mathrm{PeV}$ showers as on figure 3 are shown in figure 4 by red circles and blue circles, respectively, in comparison with the corresponding LDFs at the CORSIKA simulation level (i.e. before the detector response simulation), normalized to the composite ones, are drawn (black and green curves). In what follows we use composite LDFs to evaluate the energy of detected EAS.

Finally, we carried out trigger response simulation and evaluated the instrumental acceptance for the range of "trigger" energy $\left(E_{\text {Trig }}\right)$ of 2-200 PeV, and for a wide range of other conditions, such as the primary nucleus charge number and zenith angle. In this calculation we used noise patterns derived directly from experimental events. The trigger energy parameter was defined as $E_{\text {Trig }}=K \cdot E_{M C}$ with variable factor $K$ to provide $E_{\text {Trig }}$ in the 2-200 PeV range. For $E_{\text {Trig }}$ in the 2-17.3 PeV range the $10 \mathrm{PeV}$ CORSIKA showers were used. For the $E_{\text {Trig }}$ above $17.3 \mathrm{PeV}$ the $30 \mathrm{PeV}$ showers were used. We have verified that the $E_{M C}$ values discreteness had a limited impact on results of the simulation. Figure 5 shows the instrumental acceptance for different primary particles and $H$ around $400 \mathrm{~m}$. The acceptance for various altitudes for proton-initiated showers is shown in figure 6 . The bigger the altitude is the greater is the value of the acceptance at the highest

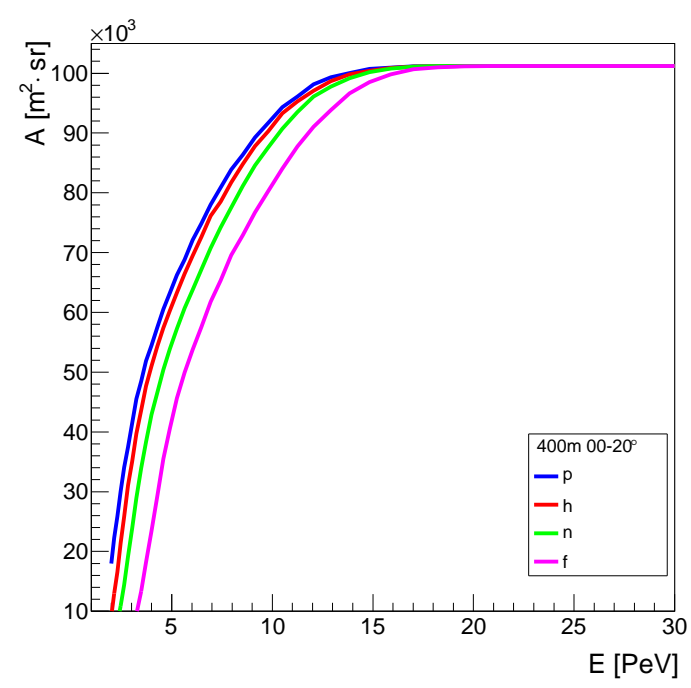

Figure 5: Acceptance for various primary nuclei mass, observation altitude $H=400 \mathrm{~m}$ a.s.l., and $\theta=$ $0-20^{\circ}$.

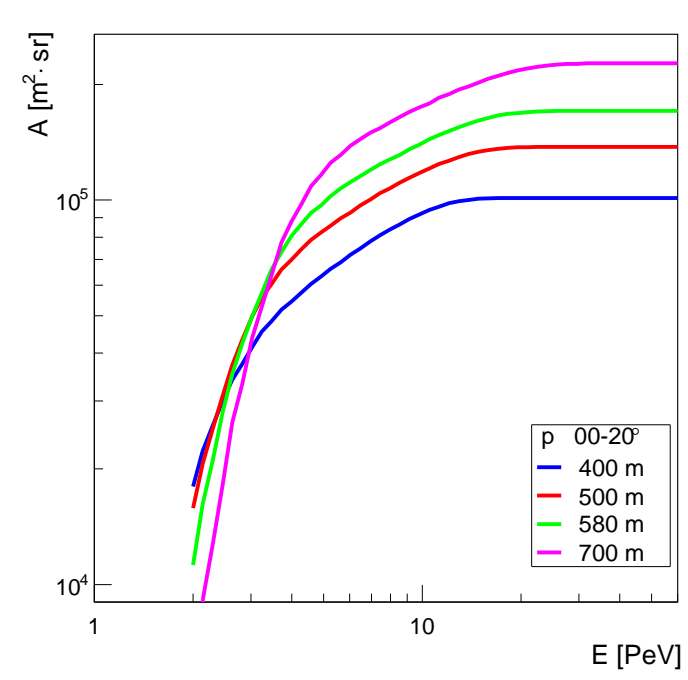

Figure 6: The same as on Figure 5 but for proton with angles $\theta=0-20^{\circ}$ and various altitude values. 
energies.

\section{Shower parameters estimation}

To estimate the energy of the primary particle the experimental LDF was fitted by a sample of composite LDFs, selecting the best-fit CRLDF for the final estimation. The normalization factor of this latter CRLDF is nearly proportional to $E_{0}$ [9]. The energy estimation procedure was tested on simulated detector response events. Figure 7 presents the energy reconstruction perfomances of this procedure for a subsample of model showers that satisfy the trigger condition. The ratios of the mean reconstructed energy to the MC true energy are shown by symbols for proton and iron primaries for the MC true primary energy values $E_{M C}$ of 10 and $30 \mathrm{PeV}$ and several altitudes $H(400,500,580,700 \mathrm{~m})$. The corresponding standard deviations are shown by bars. A small artificial shift on $H$ was introduced to make the different datasets visible. The statistical uncertainty of the primary energy estimation is from $\sim 10 \%$ to $\sim 20 \%$ depending on the primary energy and the altitude.

The systematic error does not exceed 5\% for most cases. The primary all-nuclei energy spectrum is shown on the Figure 8; its systematic uncertainties were discussed in [3]. Here we only note that these latter systematical uncertainty was found to be modest and comparable with the case of ground-based expariments such as KASCADE-Grande.

\section{Composition estimation}

The fraction of light nuclei (protons and helium) in the 10-200 PeV energy range was estimated by means of an event-by-event approach using the LDF steepness parameter $\eta$ [10].

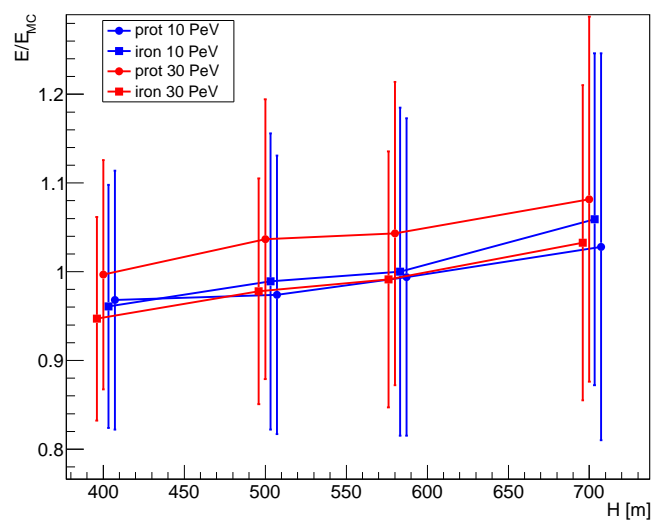

Figure 7: The ratio of the reconstructed to MC true energy for model showers with various primary nuclei mass, energy and observation altitude for showers that were registered by the model of the instrumental trigger.

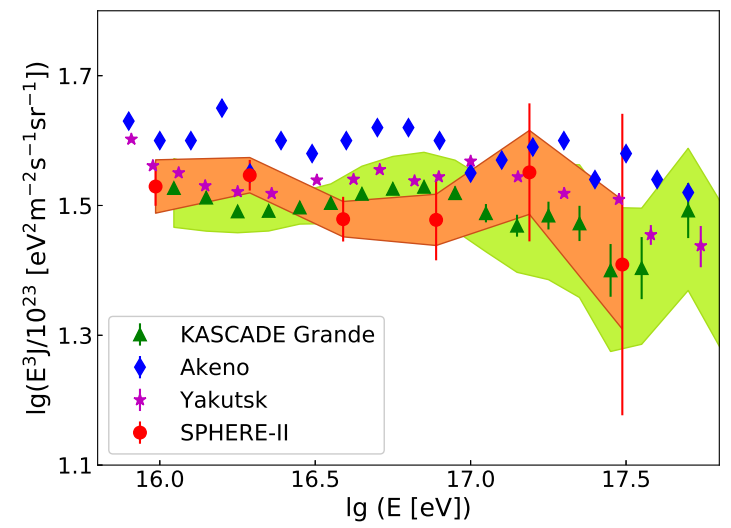

Figure 8: Energy spectrum reconstructed for the 2011-2013 runs of the SPHERE experiment. Systematical uncertainty is shown by a colored area. 


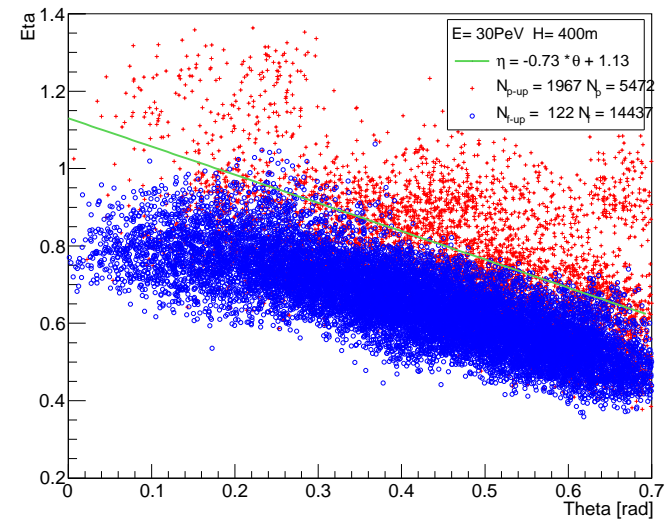

Figure 9: The result of the model showers classification for $E_{\text {Trig }}=102.6 \mathrm{PeV}$. Red crosses (protons) and blue circles (Iron) show LDF steepness parameter $\eta$ vs. $\theta$, green line is the classification border.

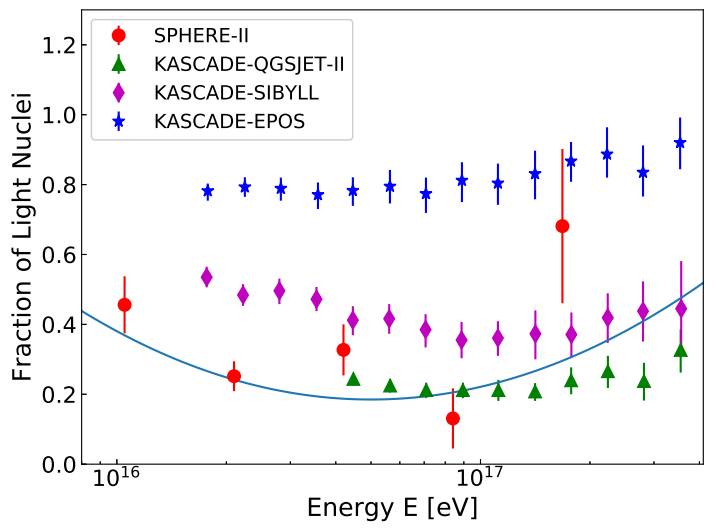

Figure 10: The fraction of light nuclei reconstructed for the 2013 run of the SPHERE experiment

The parameter $\eta$ for a shower is calculated as the ratio light collected in the ranges $0-70 \mathrm{~m}$ and 70-140 $\mathrm{m}$ from the shower axis (the fitting composite LDF integrals were used here as those have higher precision). This paramter depends on other shower conditions such as primary particle mass, energy, angle and detectors altitude. We tried to make mass $A$ determination by parameter $\eta$ for sets of other parameters $\left(E_{\text {Trig }}, \theta_{0}, H\right)$. The classifier was trained on the calculated moled LDF set. For more details see to [11].

One example of the classifier training result is shown in figure 9 for $E_{\text {Trig }}=102.6 \mathrm{PeV}$ and $H=400 \mathrm{~m}$. The values of the LDF steepness parameter for protons and iron nuclei vs $\theta_{0}$ are plotted together with the border between the classes. The numbers of the showers above the border $\left(N_{p-u p}\right.$ and $\left.N_{f-u p}\right)$, and the total numbers of these showers $\left(N_{p}\right.$ and $\left.N_{f}\right)$, respectively, are shown in the figure. Finally, the fraction of the light component $F_{L}$ in the total CR flux was reconstructedl it is shown in figure 10 together with statistical uncertanties. A smooth curve shown in the figure is a simple parametric dependence that is intended to provide a general idea of the $F_{L}$ change with energy. KASCADE-Grande results [12,13] are shown for comparison.

\section{Multidimensional method for superhigh energy CR composition study}

Earlier we had employed a set of one-dimensional criteria based on the LDF steepness parameter. Hovewer, in [11] we show that it is possible to improve the separationy of the event classes by utilizing multivariate analysis methods. In [11] we used the Bayesian multivariate pattern recognition technique assuming a multidimensional Gaussian distribution of event features [14].

Figures 11 and 12 show the fraction of Nitrogen nuclei classified as protons vs. proton selection efficiency. Black curves denote $H=400 \mathrm{~m}$, red curves $-H=580 \mathrm{~m}$, and blue curves $H=900 \mathrm{~m}$. 


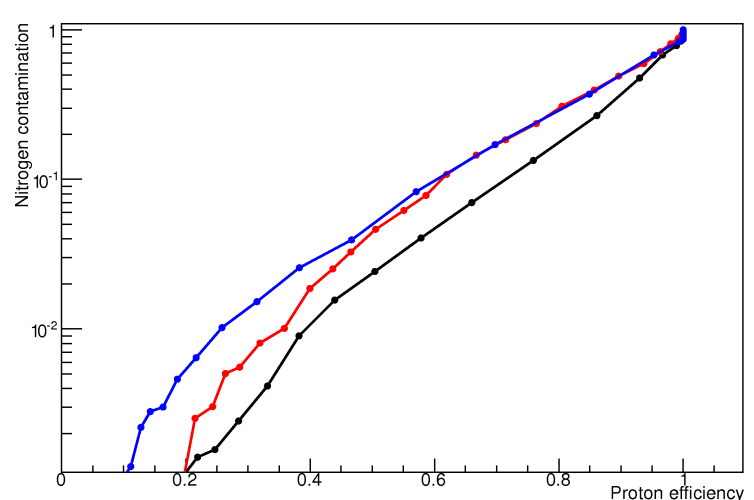

Figure 11: Proton-Nitrogen separation for $E=$ $10 \mathrm{PeV}$ and $\theta=0-20 \mathrm{deg}$

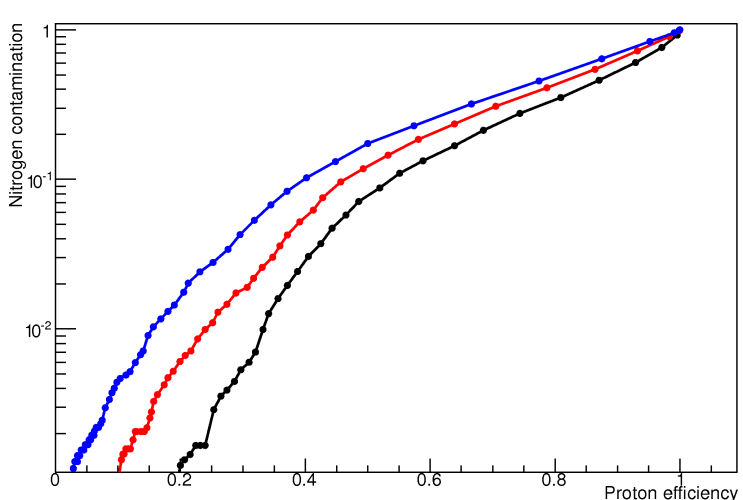

Figure 12: The same as on Figure 11 but for $\theta=$ $20-40 \mathrm{deg}$

\section{Conclutions and prospects of the method}

In this paper we have briefly reviewed the status and results of the reflected Cherenkov light method. The all-nuclei CR spectrum was reconstructed. The total uncertainty of the spectrum (including the systematical one) was shown to be comparable with the one for ground-based experiments. Moreover, we were able to reconstruct the fraction of light nuclei in CR flux using an event-to-event approach. We plan to study the dependence of these results on hadronic models in future. As well, we foresee a new experiment with a balloon-borne detector for registration of reflected CL and direct fluorescence light from EAS with energies $5 \cdot 10^{18}-5 \cdot 10^{19} \mathrm{eV}$ in Antarctica [16].

\section{References}

[1] The Pierre Auger Collaboration, The Pierre Auger Cosmic Ray Observatory, NIM A 798 (2015) $172-213$.

[2] A.E. Chudakov, The possible method of EAS registration based on Cherenkov light reflected from snow surface, in proceedings of All-USSR Symp. on Exp. Meth. of UHECR, Yakutsk (1974) 69 (in Russian).

[3] R.A. Antonov et al., Detection of Reflected Cherenkov Light from Extensive Air Showers in the SPHERE Experiment as a Method of Studying Superhigh Energy Cosmic Rays, Phys. Part. Nucl. 46 (2015) 60-93.

[4] R.A. Antonov et al., The LED calibration system of the SPHERE-2 detector, Astroparticle Physics 77 (2016) 55-65.

[5] D. Heck et al., CORSIKA: A Monte Carlo Code to Simulate Extensive Air Showers, FZKA Report 6019 (1998).

[6] N.N. Kalmykov et al., Quark-Gluon-String Model and EAS Simulation Problems at Ultra-High Energies, Nucl. Phys. Proc. Suppl. B 52 (1997) 17.

[7] H.C. Fesefeldt, Simulations of Hadronic Showers, Physics and Applications, Technical Report PITHA 85-02 RWTH (1985).

[8] S. Agostinelli et al., Geant4: a simulation toolkit, NIM A 506 (2003) 250. 
[9] L.G. Dedenko and et al., Energy estimation of inclined air showers with help of detector responses, Nucl. Phys.: Proc. Suppl. B 136 (2004) 1217.

[10] R.A. Antonov et al., A Method for primary proton spectrum measurement at $E_{o} \geq 10$ PeV with the SPHERE-2 Telescope, in proceedings of 31st ICRC, Lodz 4 (2009).

[11] R.A. Antonov et al., Event-by-event study of CR composition with the SPHERE experiment using the 2013 data, in proceedings of 24th ECRS, Kiel, Germany (2014).

[12] W.D.Apel et al., Ankle-like feature in the energy spectrum of light elements of cosmic rays observed with KASCADE-Grande Phys. Rev. D 87 (2013) 081101.

[13] W.D.Apel et al., The KASCADE-Grande energy spectrum of cosmic rays and the role of hadronic interaction models Advances in Space Research 53 (2014) 1456-1469.

[14] S. Theodoridis and K. Koutroumbas, Pattern Recognition, Elsevier, Amsterdam 2003.

[15] V.I. Galkin and T.A. Dzhatdoev, Classifying groups of PCR nuclei with energies of $10^{15}-10^{16} \mathrm{eV}$ according to the spatial-angular distribution of EAS Cherenkov light, Bulletin of the Russian Academy of Sciences: Physics 75 (2011) 338 (In Russian).

[16] D.V. Chernov et al., Detector for the ultrahigh energy cosmic rays composition study in antarctica, Journal of Physics: Conference Series 798 (2017) 1-5 